\title{
Virtual Topology Design for Minimizing Network Diameter and Average Hop Count in WDM Networks
}

\author{
Bin Wu, Kwan L. Yeung, and Pin-Han Ho
}

\begin{abstract}
We design virtual topologies in wavelength division multiplexing (WDM) networks to minimize the network diameter and average hop count, where network diameter refers to the number of hops of the longest shortest path and average hop count is the average number of hops among the shortest paths of all node pairs. Such objectives are important to WDM networks, especially to those with statistical multiplexing mechanisms such as optical burst switching (OBS) and optical packet switching (OPS). By minimizing the network diameter and average hop count, optical packets or bursts will experience less contention loss and smaller delay due to a reduced number of intermediate nodes en route. In this paper, we first formulate an integer linear program (ILP) for optimal design of virtual topologies with minimized network diameter and average hop count. Then, a novel heuristic least weight minimum diameter (LWMD) is proposed to find good solutions efficiently. Based on the virtual topology obtained, we further design two traffic accommodation schemes to provision wavelengths under a given traffic matrix, with guaranteed network diameter and minimized network resource consumption.
\end{abstract}

Index Terms-Hop count; Network diameter; Virtual topology; Wavelength division multiplexing.

\section{INTRODUCTION}

W avelength division multiplexing (WDM) technology has enabled efficient communications by combining hundreds of high-speed wavelength channels onto a single fiber for parallel data transmission [1]. Nowadays, the bandwidth of a wavelength channel can be $40 \mathrm{Gbps}$ [2] or above, whereas most subrate applications only require a bandwidth much less than this level. If the conventional circuit switching is carried out by taking the full bandwidth of a wavelength as the smallest granularity, each subrate application will engross a full wavelength capacity, which will seriously underutilize the bandwidth. Although various traffic grooming schemes [3-6] have been proposed to enable bandwidth sharing among multiple subrate applications,

Manuscript received December 9, 2009; revised August 3, 2010; accepted August 11, 2010; published November 30, 2010 (Doc. ID 121217).

Bin Wu and Pin-Han Ho are with the Department of Electrical and Computer Engineering, University of Waterloo, Waterloo, N2L 3G1, Ontario, Canada.

Kwan L. Yeung (e-mail: kyeung@eee.hku.hk) is with the Department of Electrical and Electronic Engineering, The University of Hong Kong, Pokfulam, Hong Kong.

Digital Object Identifier 10.1364/JOCN.2.001077 bandwidth utilization is still limited by the circuit-switching nature of those schemes.

To achieve higher bandwidth utilization, optical burst switching (OBS) [7-14] and optical packet switching (OPS) [14-17] have been proposed to leverage statistical multiplexing. OPS can achieve the highest bandwidth utilization, but it requires fast optical switching and optical buffer technologies, which are still far from reality [15]. OBS is based on more mature optical technologies. It relies on a one-way resource reservation scheme for contention resolution, but its performance is greatly affected by burst contentions in the network.

In OPS- and OBS-based WDM networks, network diameter and average hop counts are two important performance metrics. Network diameter is defined as the hop count of the shortest path between the two furthest apart nodes in the network, or the hop count of the longest shortest path. Average hop count is the average number of hops of all shortest paths, where each shortest path is found between a distinct node pair and all node pairs in the network are taken into account.

To illustrate the importance of network diameter and average hop count, we consider the hybrid OBS switching paradigm proposed in [12], where in-band signaling is adopted. A control packet is sent ahead of the data burst for wavelength reservation. After a predetermined offset time, the data burst is sent along the same route (i.e., in-band signaling) without knowing the result of the wavelength reservation. If the control packet can successfully reserve a wavelength at each and every hop, an optical path will be scheduled to transmit the data burst all-optically to the destination. Otherwise, both the control packet and the subsequently arrived data burst will be dropped at an intermediate node due to reservation failure. Although the data burst is transmitted all-optically, the control packet must be electrically processed at each hop. To support such opticalelectrical hybrid switching, by default each intermediate node works in an O-E-O mode for receiving and processing the control packet. A successful reservation by the control packet at an intermediate node will schedule a period of time for the subsequent all-optical transmission of the data burst. The offset time (between sending the control packet and the data burst) must be sufficiently large to allow control packet processing at all intermediate nodes. If the (OE-O) hop count of the connection can be reduced, the loss of both the control packet and data burst due to contention will 
be reduced, and this also helps to minimize the traffic delay caused by the offset time. Similarly, in the OPS scenario, if the hop count of the connection can be reduced, optical packets will encounter less contention, and the performance of the OPS network can also be improved.

In WDM networks, minimizing connection hop counts can be achieved by virtual topology design [12,18-24]. Specifically, we can pick up a wavelength channel from each of a set of consecutive fiber links and connect them to form a lightpath [19], where optical signals can traverse the lightpath transparently (and without O-E-O conversion). Effectively, the lightpath becomes a single virtual hop/link. Without loss of generality, we assume that a lightpath uses the same wavelength on each fiber link.

In this paper, we focus on virtual topology design that aims at minimizing the network diameter as the primary objective and average hop count as the secondary objective. Note that the network diameter is the maximum hop-count limit for all connections (if shortest-path routing is adopted). Although virtual topology design has been extensively studied in the literature [12,18-24], we only found two papers $[12,18]$ on minimizing the network diameter. Notably, the work in [18] only considers linear and ring physical topologies, and the work in [12] adopts the simulated annealing technique [25] to minimize the network diameter in optical mesh networks. Neither of them directly addresses the issue of minimizing the average hop count.

We take both the network diameter and average hop count into account. An integer linear program (ILP) for optimal virtual topology design is first formulated. To avoid the huge computational complexity of the ILP, we propose an efficient heuristic least weight minimum diameter (LWMD). Unlike the randomized simulated annealing approach in [12], LWMD systematically constructs virtual topologies in a deterministic manner. Because WDM networks do not necessarily have bidirectional links, we focus on general WDM networks with unidirectional links. Based on virtual topologies generated by LWMD, two traffic accommodation schemes are proposed to provision wavelengths for a given traffic matrix. Our objective is to keep the same network diameter while minimizing network resource consumption.

The rest of the paper is organized as follows. Section II formulates the ILP for optimal virtual topology design. Section III details the LWMD mechanism and presents some numerical results. The two traffic accommodation schemes are proposed in Section IV, and we conclude the paper in Section V.

\section{ILP FORMULATION}

\section{A. General Idea}

Since we want to minimize both the network diameter and average hop count, we can denote the objective function of the ILP as a linear weighted sum of the two parameters. There are two key issues in the ILP: 1 ) we need to formulate how virtual links are constructed in the physical topology such that the ILP can search for the optimal solution in its optimization process, and 2) based on the virtual topology yet to be determined, we need to formulate all-pairs shortest paths to determine the network diameter and the hop count of the shortest path between any pair of nodes.

We adopt the flow conservation property [26] of a virtual link to solve issue 1 . If there is a virtual link from node $x$ to node $y$, then $x$ must have an outgoing wavelength channel for this virtual link and $y$ must have an incoming wavelength channel. Meanwhile, all other nodes in the network must have an equal number of incoming and outgoing wavelength channels for this virtual link.

To address issue 2, we use Floyd-Warshall algorithm [26]. Floyd-Warshall algorithm is a very efficient iterative algorithm for calculating the shortest paths of all the node pairs in the network, and its pseudocode is shown in Fig. 1.

\section{B. Notation List}

$D \quad$ General integer variable. It is the network diameter.

$d_{x y}^{k} \quad$ General integer variable. It is the hop count of the shortest path from node $x$ to node $y$ in the $k$ th iteration.

$g_{x y}^{k} \quad$ Binary variable. It takes 1 if $d_{x y}^{k}>d_{x k}^{k}+d_{k y}^{k}$, and 0 otherwise.

$v_{x y} \quad$ Binary variable. It takes 1 if there is a virtual link from node $x$ to node $y$, and 0 otherwise.

$p_{x y}^{i j} \quad$ Binary variable. It takes 1 if there is a virtual link from node $x$ to node $y$, and this virtual link traverses directed fiber link $(i, j)$ from node $i$ to node $j$. Otherwise it is 0 .

$W \quad$ Predefined constant. It is the number of available wavelengths on each fiber link.

$I \quad$ Predefined constant. It is an integer large enough ( $I$ $\gg D$ ) to represent the infinite distance of two nodes in the initialization step of Floyd-Warshall algorithm (see Fig. 1), if the two nodes are not directly connected by a link.

$C \quad$ Predefined constant and $C \gg I$.

$\beta \quad$ Predefined constant. It is the weighting factor between the network diameter and average hop count in the objective function of the ILP. Its value can be determined according to the particular engineering design emphasis.

$\boldsymbol{N}$ The set of all the $N$ nodes $\{0,1,2, \ldots, N-1\}$ in the network.

$\boldsymbol{E}$ The set of all the directed links in the physical topology.

\section{Flovd-Warshall Algorithm}

$\operatorname{dist}(i, j)$ : the hop count of the shortest path from node $i$ to node $j$. $\operatorname{cost}(i, j)$ : the cost of link $(i, j)$, or $\infty$ if no link from node $i$ to node $N$ : the number of a set of nodes $\left\{0,1,2, \ldots, N^{-1}\right\}$.

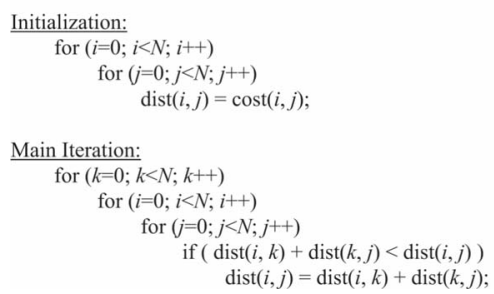

Fig. 1. Pseudocode of Floyd-Warshall algorithm. 


\section{ILP Formulation}

Objective:

$$
\operatorname{minimize}\left\{D+\beta \sum_{x \in \boldsymbol{N}} \sum_{y \in \boldsymbol{N}: y \neq x} d_{x y}^{N}\right\}
$$

Subject to:

$$
\begin{aligned}
& \sum_{(x, j) \in \boldsymbol{E}} p_{x y}^{x j}=v_{x y}, \quad \forall x, y \in \mathbf{N}: x \neq y \\
& \sum_{(i, y) \in \boldsymbol{E}} p_{x y}^{i y}=v_{x y}, \quad \forall x, y \in \boldsymbol{N}: x \neq y \\
& \sum_{(i, j) \in \boldsymbol{E}} p_{x y}^{i j}=\sum_{(j, i) \in \boldsymbol{E}} p_{x y}^{j i}, \quad \forall x, y \in \boldsymbol{N}: x \neq y, \\
& \forall j \in \boldsymbol{N}: j \neq x, y \text {; } \\
& \sum_{x \in \boldsymbol{N}} \sum_{y \in \boldsymbol{N}: y \neq x} p_{x y}^{i j}=W, \quad \forall(i, j) \in \boldsymbol{E} ; \\
& d_{x y}^{0}=I\left(1-v_{x y}\right)+v_{x y}, \quad \forall x, y \in \mathbf{N}: x \neq y \\
& d_{x x}^{0}=0, \quad \forall x \in \mathbf{N} ; \\
& d_{x y}^{k+1} \leqslant d_{x y}^{k}, \quad \forall x, y, k \in \boldsymbol{N} \\
& d_{x y}^{k+1} \leqslant d_{x k}^{k}+d_{k y}^{k}, \quad \forall x, y, k \in \boldsymbol{N} \\
& g_{x y}^{k} \geqslant \frac{1}{C}\left(d_{x y}^{k}-d_{x k}^{k}-d_{k y}^{k}\right), \quad \forall x, y, k \in \mathbf{N} ; \\
& g_{x y}^{k} \leqslant 1-\frac{1}{C}\left(d_{x k}^{k}+d_{k y}^{k}-d_{x y}^{k}\right), \quad \forall x, y, k \in \mathbf{N} ; \\
& d_{x y}^{k+1} \geqslant d_{x k}^{k}+d_{k y}^{k}-C\left(1-g_{x y}^{k}\right), \quad \forall x, y, k \in \boldsymbol{N} ; \\
& d_{x y}^{k+1} \geqslant d_{x y}^{k}-C g_{x y}^{k}, \quad \forall x, y, k \in \boldsymbol{N} ; \\
& D \geqslant d_{x y}^{N}, \quad \forall x, y \in \boldsymbol{N} .
\end{aligned}
$$

Objective (1) minimizes a linear weighted sum of the network diameter and the total hop count. The set of constraints (2)-(5) are used to formulate the virtual links, and the remaining constraints (6)-(14) are used to implement Floyd-Warshall algorithm.

Focusing on virtual link formulation, constraints (2)-(4) specify the flow conservation property of a virtual link. In particular, constraints (2) and (3) say that if there is a virtual link from node $x$ to node $y$, then it must have an outgoing wavelength channel at $x$ and an incoming wavelength channel at $y$. Constraint (4) requires that all other nodes in the network must obey flow conservation for those wavelength channels used to implement this virtual link. Constraint (5) means that the total number of wavelength chan-

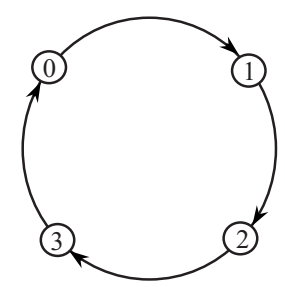

(a) Ring physical topology.

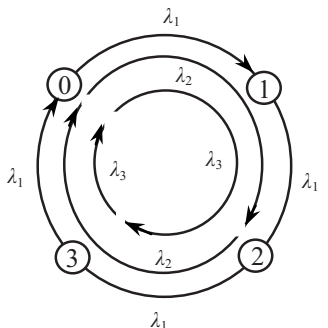

(b) Virtual topology with a diameter of 2 .
Fig. 2. Optimal ILP solution for a ring topology with $N=4, W=3$, and $\beta=0$.

nels on each link is $W$, which can be used to implement those virtual links passing through this physical link.

To implement Floyd-Warshall algorithm, constraints (6) and (7) are the initialization steps (see Fig. 1) based on the virtual topology (yet to be determined by the optimization process). If there is a virtual link from node $x$ to node $y$, then the initial distance $d_{x y}^{0}$ is set to 1 (hop) and otherwise $d_{x y}^{0}$ $=\infty($ represented by $I)$. A node has a 0 distance to itself according to constraint (7). Constraints (8) and (9) bound the distance $d_{x y}^{k+1}$ in the $(k+1)$ th iteration using the result $\min \left(d_{x y}^{k}, d_{x k}^{k}+d_{k y}^{k}\right)$ obtained in the $k$ th iteration. Constraints (10) and (11) define $g_{x y}^{k}$, which takes 1 if $d_{x y}^{k}>d_{x k}^{k}+d_{k y}^{k}$ and 0 otherwise. By working with constraints (8)-(11), constraints (12) and (13) further ensure that $d_{x y}^{k+1}=\min \left(d_{x y}^{k}, d_{x k}^{k}+d_{k y}^{k}\right)$, which properly implements the main iteration of FloydWarshall algorithm. Finally, constraint (14) finds the network diameter $D$ from the hop counts of the all-pairs shortest paths saved in $d_{x y}^{N}$.

\section{An Example}

Figure 2(b) shows an optimal ILP solution for the fournode ring topology in Fig. 2(a) with three wavelengths on each link, where we set $\beta=0$ in objective function (1) to minimize the network diameter only. Compared with the physical topology in Fig. 2(a) with a network diameter of 3 and an average hop count of 2, the virtual topology in Fig. 2(b) has a network diameter of 2 and an average hop count of 1.5. If we set $\beta=1$ in objective (1), we can get another optimal solution with a network diameter of 2 and an average hop count of 1.33 . In general, $\beta$ can be used to manipulate the weights of the two minimization objectives in objective function (1), and its value can be determined according to the emphasis of particular engineering designs.

\section{LWMD ALGORITHM}

Solving the ILP in contraints (1)-(14) is too time consuming. In this section, we propose an efficient heuristic LWMD to minimize both the network diameter and average hop count.

\section{A. General Idea}

In LWMD, each of the available wavelengths $\left\{\lambda_{i} \mid W \geqslant i\right.$ $\geqslant 1\}$ is sequentially used to construct the virtual topology. We use current topology $\boldsymbol{V}$ to represent the virtual topology 
constructed so far. Each edge in $\boldsymbol{V}$ is a virtual link that corresponds to a virtual hop. The virtual link is implemented by pre-cross-connecting the same wavelength on several contiguous physical links. For simplicity, we say that a virtual link is over $\lambda_{i}$ if it is operated at $\lambda_{i}$. In such a virtual topology, an all-optical path may consist of several virtual links over different wavelengths, and thus wavelength converters may be required to connect those virtual links.

The current topology $\boldsymbol{V}$ is first initialized to the same as the physical topology, with each virtual link over $\lambda_{1}$. In other words, $\lambda_{1}$ is used to ensure the same connectivity as in the physical topology. Then we sequentially use $\lambda_{2}-\lambda_{W}$ to improve the current topology $\boldsymbol{V}$, with the goal of minimizing the network diameter and the average hop count. This process is called wavelength scanning. By default, we treat $\lambda_{1}$ as an already-scanned wavelength.

Without loss of generality, we consider the scanning process of $\lambda_{s}(W \geqslant s \geqslant 2)$. First, we $a d d \lambda_{s}$ to $\boldsymbol{V}$. This is done by translating each physical link into a virtual link over $\lambda_{s}$ and then adding the virtual link to $\boldsymbol{V}$. In essence, it means that at the beginning each physical link is treated as a virtual link over $\lambda_{s}$.

After $\lambda_{s}$ is added, LWMD constructs a set of tentative topologies from $\boldsymbol{V}$, among which the best one is used to update/ improve $\boldsymbol{V}$. Define the longest shortest path (LSP) as the path with the largest hop count among the all-pairs shortest paths [26]. To construct the set of tentative topologies, we first find all LSPs in $\boldsymbol{V}$. Each tentative topology $\boldsymbol{V}^{\prime}$ is obtained from $\boldsymbol{V}$ by merging two adjacent virtual links on an LSP to form a single virtual link over $\lambda_{s}$. This can reduce the (virtual) hop count of the target LSP by 1 . Then a set of tentative topologies can be obtained by considering all possible mergings on each LSP. The merging process is detailed in the next subsection.

For a tentative topology $\boldsymbol{V}^{\prime}$, we define the total (shortest path) hop distance between all node pairs in $\boldsymbol{V}^{\prime}$ as its weight, or

$$
W\left(\boldsymbol{V}^{\prime}\right)=\sum_{i, j \in \boldsymbol{V}^{\prime}} h_{i j},
$$

where $h_{i j}$ is the (virtual) hop count of the shortest path from node $i$ to node $j$. When the weight is divided by the number of node pairs, we get the average hop count of the shortest paths. In other words, minimizing weight is the same as minimizing the average hop count.

Among all the tentative topologies $\boldsymbol{V}^{\prime}$ obtained, the one with the least weight is chosen to update $\boldsymbol{V}$, provided that both its diameter and weight are not larger than that of $\boldsymbol{V}$. Based on the updated current topology $\boldsymbol{V}$, we identify a new set of LSPs and repeat the above process to find another $\boldsymbol{V}^{\prime}$ to improve $\boldsymbol{V}$. The $\lambda_{s}$ scanning process ends when $\boldsymbol{V}$ cannot be further improved. That is, each and every obtained tentative topology $\boldsymbol{V}^{\prime}$ has either a larger diameter or a larger weight than $\boldsymbol{V}$. At this point, we say that $\lambda_{s}$ is exhausted, and we proceed to scan the next wavelength. The final solution is reached when all $\lambda_{2}-\lambda_{W}$ have been scanned and exhausted.

\section{B. Merging for Tentative Topology Construction}

The key issue in the LWMD algorithm is to construct the set of tentative topologies $\boldsymbol{V}^{\prime}$. Assume $\lambda_{s}$ is being scanned, and two adjacent virtual links $a \rightarrow b$ and $b \rightarrow c$ on an LSP are to be merged to construct a new $\boldsymbol{V}^{\prime}$. If both virtual links are over the same wavelength $\lambda_{s}$, we can simply replace them by a new virtual link $a \rightarrow c$ over $\lambda_{s}$, i.e., $\lambda_{s}$ on $a \rightarrow b$ and $b \rightarrow c$ are used to construct the new virtual link $a \rightarrow c$.

If the two virtual links are over different wavelengths, the merging process is more complex. We first assume that virtual link $a \rightarrow b$ is over $\lambda_{s}$, and $b \rightarrow c$ is over another (previously scanned) wavelength $\lambda_{i}(s>i \geqslant 1)$. In this case, the two virtual links cannot be directly merged using $\lambda_{s}$. This is because $b \rightarrow c$ (over $\lambda_{i}$ ) was established during the previous $\lambda_{i}$ scanning, and it must remain in $\boldsymbol{V}^{\prime}$. If $b \rightarrow c$ (over $\lambda_{i}$ ) is replaced/removed during the current $\lambda_{s}$ scanning, then the reduction in network diameter and weight achieved by previous scanning (if any) cannot be honored.

Our approach is to find an alternative path $\boldsymbol{P}_{\boldsymbol{b c}}$ (over $\lambda_{s}$ ) from node $b$ to node $c$ in $\boldsymbol{V}$ and merge $\boldsymbol{P}_{\boldsymbol{b c}}$ (instead of virtual link $b \rightarrow c$ over $\lambda_{i}$ ) with $a \rightarrow b$. $\boldsymbol{P}_{\boldsymbol{b c}}$ must only consist of intact virtual links over $\lambda_{s}$, which have never been involved in any merging process since $\lambda_{s}$ was added to $\boldsymbol{V}$. In other words, each intact virtual link corresponds to a physical link. Due to this property, we call $\boldsymbol{P}_{\boldsymbol{b c}}$ an alternative physical path (APP). If multiple APPs exist, the shortest one is chosen, and is merged with $a \rightarrow b$ to form a new virtual link $a \rightarrow c$ over $\lambda_{s}$. A tentative topology $\boldsymbol{V}^{\prime}$ is thus obtained by adding $a \rightarrow c$ (over $\lambda_{s}$ ) to $\boldsymbol{V}$. At the same time, the old $a \rightarrow b$ (over $\lambda_{s}$ ) and all the intact virtual links (over $\lambda_{s}$ ) along $\boldsymbol{P}_{\boldsymbol{b c}}$ are removed.

Next we consider the case that the two virtual links to be merged are over two previously scanned wavelengths. Without loss of generality, we assume that virtual links $a \rightarrow b$ and $b \rightarrow c$ are over $\lambda_{i}$ and $\lambda_{j}$, respectively, and $s>i>j>1$. In this case, we find two APPs over $\lambda_{s}, \boldsymbol{P}_{\boldsymbol{a b}}$ from $a$ to $b$, and $\boldsymbol{P}_{\boldsymbol{b c}}$ from $b$ to $c$. We then merge $\boldsymbol{P}_{\boldsymbol{a} \boldsymbol{b}}$ and $\boldsymbol{P}_{\boldsymbol{b c}}$ to form a new virtual link $a \rightarrow c$ over $\lambda_{s}$, such that the hop count from $a$ to $c$ can be reduced from 2 to 1 . The new virtual link $a \rightarrow c$ (over $\lambda_{s}$ ) is added to $\boldsymbol{V}^{\prime}$. All intact virtual links over $\lambda_{s}$ along $\boldsymbol{P}_{\boldsymbol{a} \boldsymbol{b}}$ and $\boldsymbol{P}_{\boldsymbol{b c}}$ are removed, but the original $a \rightarrow b$ (over $\lambda_{i}$ ) and $b$ $\rightarrow c$ (over $\lambda_{j}$ ) remain.

However, we may not always be able to find a feasible APP $\boldsymbol{P}_{\boldsymbol{a} \boldsymbol{b}}$ or $\boldsymbol{P}_{\boldsymbol{b c}}$. When this happens, we give up the current effort of constructing $\boldsymbol{V}^{\prime}$ by merging $a \rightarrow b$ and $b \rightarrow c$.

To summarize, in $\lambda_{s}$ scanning, if a virtual link to be merged for $\boldsymbol{V}^{\prime}$ construction is over $\lambda_{s}$, then it is removed from $\boldsymbol{V}^{\prime}$ after the merging. If it is over a wavelength scanned earlier (i.e., among $\lambda_{1}-\lambda_{s-1}$ ), then it remains in $\boldsymbol{V}^{\prime}$ after the merging. In the latter case, we check whether there are some feasible APPs between the two end nodes of the virtual link. If yes, then the shortest APP among them is used for the current merging, and all the intact virtual links of the chosen APP are removed. Otherwise, we skip the current merging. The new virtual link (over $\lambda_{s}$ ) obtained from the merging is always added to $\boldsymbol{V}^{\prime}$.

Figure 3 shows a simple example based on the same ring topology as in Fig. 2, with $N=4$ nodes and $W=3$ wavelengths. In Fig. 3(a), $\boldsymbol{V}$ is initialized to the same as the 


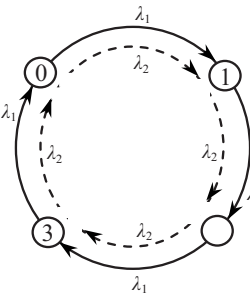

(a)

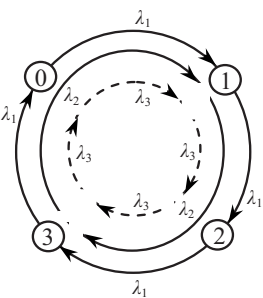

(b)

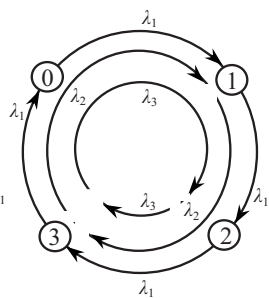

(c)
Fig. 3. LWMD design for a ring topology with $N=4$ and $W=3$.

physical topology using $\lambda_{1}$, and $\lambda_{2}$ is then added to $\boldsymbol{V}$ as indicated by the four dashed arrows. Figure $3(\mathrm{~b})$ gives the result of $\lambda_{2}$ scanning, where $3 \rightarrow 0$ and $0 \rightarrow 1$ in Fig. 3(a) are merged to form a new virtual link $3 \rightarrow 1$ over $\lambda_{2}$, and $1 \rightarrow 2$ and $2 \rightarrow 3$ are merged to form $1 \rightarrow 3$ over $\lambda_{2}$. The original virtual links $3 \rightarrow 0,0 \rightarrow 1,1 \rightarrow 2$, and $2 \rightarrow 3$ (over $\lambda_{2}$ ) are removed. Then, $\lambda_{3}$ is added to the new $\boldsymbol{V}$ as shown by the four dashed arrows in Fig. 3(b). In $\lambda_{3}$ scanning, two virtual links $1 \rightarrow 2$ (over $\lambda_{3}$ ) and $3 \rightarrow 1$ (over $\lambda_{2}$ ) in Fig. 3(b) are merged. Since the latter is over $\lambda_{2}$ instead of $\lambda_{3}$, a feasible APP 3 $\rightarrow 0 \rightarrow 1$ is found from node 3 to node 1 , which consists of (dashed) virtual links $3 \rightarrow 0$ and $0 \rightarrow 1$ over $\lambda_{3}$ [see Fig. 3(b)]. This APP is merged with $1 \rightarrow 2$ (over $\lambda_{3}$ ) to form a new virtual link $3 \rightarrow 2$ over $\lambda_{3}$, as shown in Fig. 3(c). Note that the three original virtual links over $\lambda_{3}$ [i.e., the dashed $3 \rightarrow 0$, $0 \rightarrow 1$, and $1 \rightarrow 2$ in Fig. 3(b)] are all removed, whereas 3 $\rightarrow 1$ over $\lambda_{2}$ remains in Fig. 3(c). At this point, it is not possible to further merge $2 \rightarrow 3$ (over $\lambda_{3}$ ) with $3 \rightarrow 1$ (over $\lambda_{2}$ ) in Fig. 3(c) because no feasible APP can be found for $3 \rightarrow 1$ (over $\lambda_{2}$ ). From Fig. 3(c), we can see that the network diameter is reduced from 3 [in Fig. 3(a)] to 2, and the average hop count is reduced from 2 to 1.42 . The results are comparable to the optimal ILP results obtained in Subsection II.D. In addition, the number of one-hop connections increases from 4 to 7 .

\section{Implementation Issues}

The LWMD algorithm is summarized by the flow chart in Fig. 4, and the $\mathrm{C}$ source code can be found at [27]. We use an adjacency matrix $\boldsymbol{P}=\left\{p_{i j}\right\}$ to represent the physical topology, where $p_{i j}=1$ if node $i$ is connected to node $j$ by a physical link, and $p_{i j}=\infty$ otherwise. For each wavelength $\left(\lambda_{s}\right)$ scanning, a matrix $\boldsymbol{L}=\left\{l_{i j}\right\}$ is used to record all the current virtual links over $\lambda_{s}$. When $\lambda_{s}$ is added to $\boldsymbol{V}, \boldsymbol{L}$ is initialized to represent the physical topology, where

$$
l_{i j}=\left\{\begin{array}{ll}
1 & \text { if } p_{i j}=1 \\
0 & \text { otherwise }
\end{array} .\right.
$$

In $\lambda_{s}$ scanning, $\boldsymbol{L}$ becomes

$$
\left\{\begin{array}{ll}
l_{i j}=m \geqslant 1: & i \rightarrow j \text { is a virtual link over } \lambda_{s} \\
& \text { with } m \text { hops in physical links } \\
l_{i j}=0: & i \rightarrow j \text { is not a virtual link over } \lambda_{s}
\end{array} .\right.
$$

By referring to $\boldsymbol{L}$, we can easily identify whether or not a virtual link in $\boldsymbol{V}$ is over $\lambda_{s}$.

Assume that a proper $\boldsymbol{V}^{\prime}$ for updating $\boldsymbol{V}$ is obtained by merging two virtual links $a \rightarrow b$ and $b \rightarrow c$. When $\boldsymbol{V}$ is

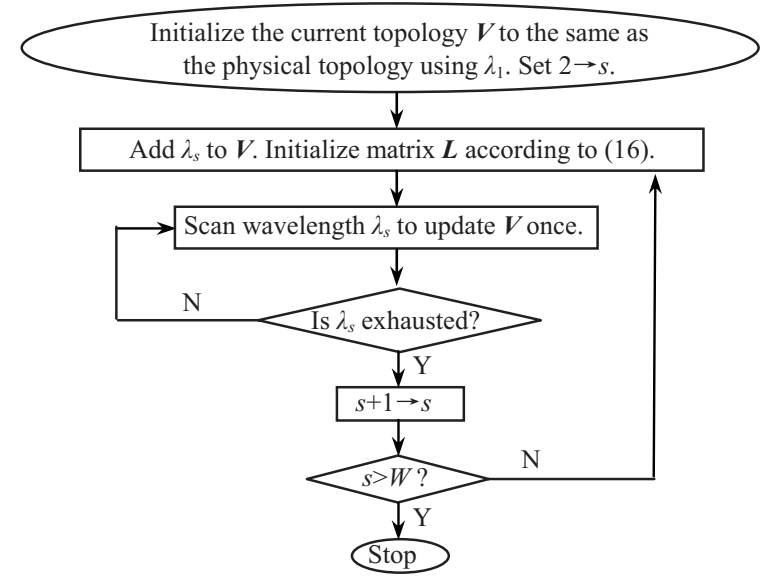

(a) Main flow

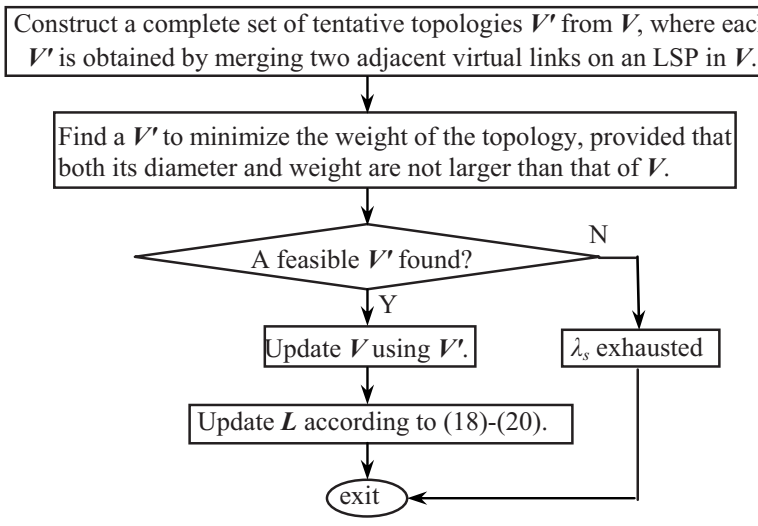

(b) Scanning $\lambda_{s}$ to update $\boldsymbol{V}$ once

Fig. 4. LWMD flow chart.

updated, we also update $\boldsymbol{L}$ according to the three cases below.

Case 1: Both $a \rightarrow b$ and $b \rightarrow c$ are over $\lambda_{s}$. In this case, both $l_{a b}$ and $l_{b c}$ are greater than 0 according to Eq. (17). $\boldsymbol{L}$ is updated by

$$
\left\{\begin{array}{c}
l_{a c}=l_{a b}+l_{b c} \\
0 \rightarrow l_{a b} \\
0 \rightarrow l_{b c}
\end{array}\right.
$$

Case 2: One virtual link (say $b \rightarrow c$ ) is over $\lambda_{s}$, and the other one $(a \rightarrow b)$ is over $\lambda_{i}(s>i \geqslant 1)$. From Eq. (17), $l_{a b}=0$ and $l_{b c} \geqslant 1$. We try to find a feasible APP $\boldsymbol{P}_{\boldsymbol{a} \boldsymbol{b}}$ for merging. Specifically, we pick up all the entries $\left\{(i, j) \in \boldsymbol{L} \mid l_{i j}=1\right\}$ from $\boldsymbol{L}$ to construct a residual topology, which is a subgraph of the physical topology. Each of these entries corresponds to a link in the residual topology. $l_{i j}=1$ means that $\lambda_{s}$ on link $i$ $\rightarrow j$ is intact or never involved in any previous merging after $\lambda_{s}$ was added to $\boldsymbol{V}$ [see Eqs. (16) and (17)]. In the residual topology, a shortest path $\boldsymbol{P}_{\boldsymbol{a} \boldsymbol{b}}$ from node $a$ to node $b$ is found by Dijkstra's algorithm [26] and is used for the current merging. Let $i \rightarrow j$ be an intact virtual link on $\boldsymbol{P}_{\boldsymbol{a} \boldsymbol{b}} . \boldsymbol{L}$ is then updated by 


$$
\left\{\begin{array}{c}
l_{a b}=\sum_{(i \rightarrow j) \in \boldsymbol{P}_{\boldsymbol{a b}}} l_{i j} \\
l_{a c}=l_{a b}+l_{b c} \\
\left.0 \rightarrow l_{i j}\right|_{(i \rightarrow j) \in \boldsymbol{P}_{\boldsymbol{a b}}} \\
0 \rightarrow l_{a b} \\
0 \rightarrow l_{b c}
\end{array}\right.
$$

Case 3: Both $a \rightarrow b$ and $b \rightarrow c$ are over a previously scanned wavelength. From Eq. (17), both $l_{a b}$ and $l_{b c}$ equal 0 . We use the same mechanism as in Case 2 to get the residual topology, as well as the two feasible APPs $\boldsymbol{P}_{\boldsymbol{a} \boldsymbol{b}}$ and $\boldsymbol{P}_{\boldsymbol{b c}}$. Then, $\boldsymbol{L}$ is updated by

$$
\left\{\begin{array}{c}
l_{a b}=\sum_{(i \rightarrow j) \in \boldsymbol{P}_{\boldsymbol{a} \boldsymbol{b}}} l_{i j}, \quad l_{b c}=\sum_{(i \rightarrow j) \in \boldsymbol{P}_{\boldsymbol{b c}}} l_{i j} \\
l_{a c}=l_{a b}+l_{b c} \\
\left.0 \rightarrow l_{i j}\right|_{(i \rightarrow j) \in \boldsymbol{P}_{\boldsymbol{a b}}},\left.\quad 0 \rightarrow l_{i j}\right|_{(i \rightarrow j) \in \boldsymbol{P}_{\boldsymbol{b c}}} \\
0 \rightarrow l_{a b} \\
0 \rightarrow l_{b c}
\end{array}\right.
$$

From Eqs. (18)-(20), we can see no matter how $\boldsymbol{L}$ is updated, the conditions specified in Eq. (17) are always satisfied. Note that $\boldsymbol{L}$ is referred to in the constructions of both the tentative topology and the residual topology.

\section{Time Complexity}

Let $N$ be the number of nodes in the network and $W$ be the number of available wavelengths for the LWMD design. Assume that the maximum node degree is $D$. Such a network contains at most $O(N D)$ physical links. In each $\lambda_{s}$ scanning, each physical link is first translated into a virtual link over $\lambda_{s}$ in $\boldsymbol{V}$. Then LWMD merges some pairs of adjacent virtual links on an LSP to reduce the diameter and the weight of the topology. There are at most $O(N D W)$ virtual links in $\boldsymbol{V}$. In the worst case, LWMD needs to construct a tentative topology $\boldsymbol{V}^{\prime}$ by merging each of these virtual links. With Floyd-Warshall algorithm [26] for finding the weight $W\left(\boldsymbol{V}^{\prime}\right)$, this gives a complexity of $O\left(D W N^{4}\right)$. Then LWMD will find a $\boldsymbol{V}^{\prime}$ to update $\boldsymbol{V}$. Before carrying out each merging, Dijkstra's algorithm may be executed in the residual topology to check the feasibility of merging, but it does not dominate the time complexity of the algorithm. When adding $\lambda_{s}$ to $\boldsymbol{V}$, there are at most $O(N D)$ virtual links over $\lambda_{s}$. Since each update of $\boldsymbol{V}$ is caused by merging at least two virtual links over $\lambda_{s}, \lambda_{s}$ is exhausted by at most $O(N D)$ updates. Because LWMD needs to scan $O(W)$ wavelengths, the total time complexity of the LWMD algorithm is $O\left(D^{2} W^{2} N^{5}\right)$.

\section{E. Performance and Design Examples}

Figures 5 and 6 show the performance of LWMD based on randomly generated physical topologies. The outgoing degree of each node is randomly selected from a discrete uniform distribution $\{1,2, \ldots, D\}$. In Fig. 5 , we set $N=50$ and $W=10$. $D$ is increased from 2 to 8 . For each $D$, we average the results over 100 topologies. From the figure, we can see that both the diameter and the average hop count are significantly reduced, especially when the network degree/

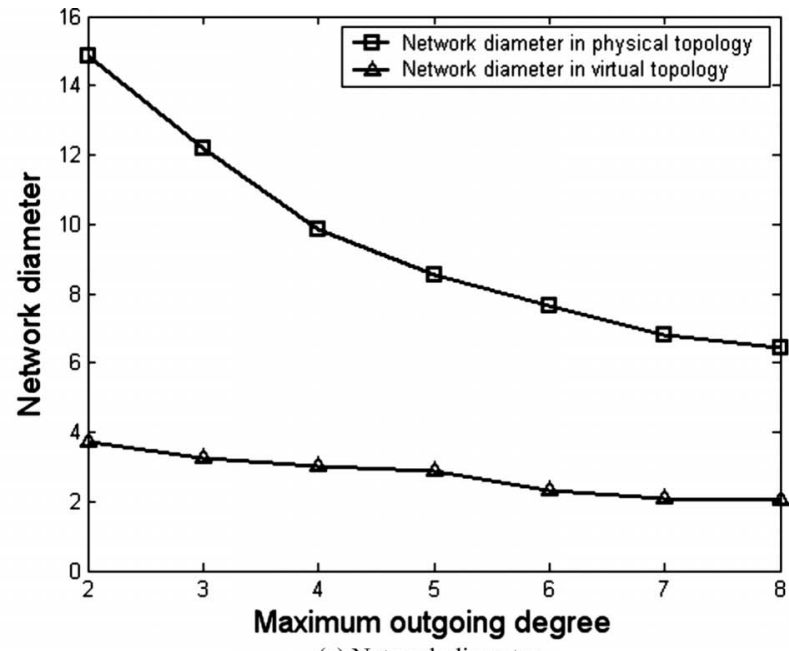

(a) Network diameter.

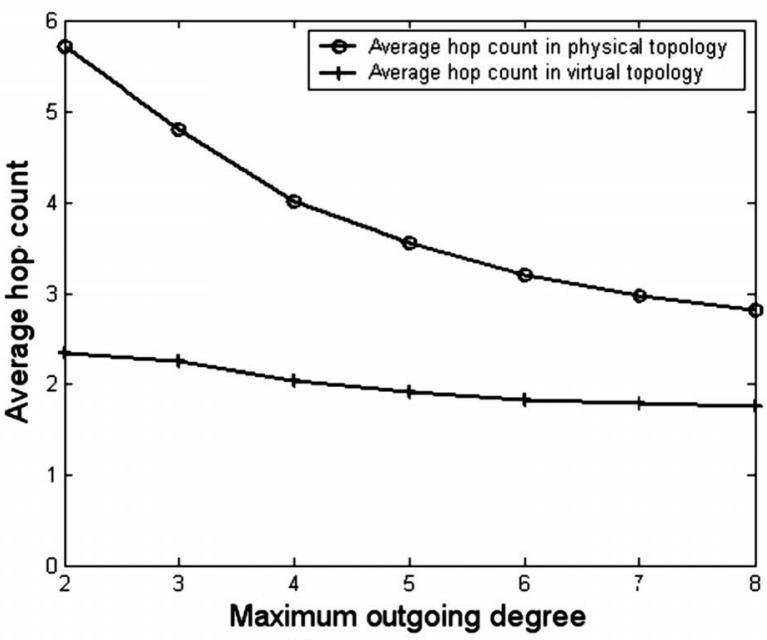

(b) Average hop count.

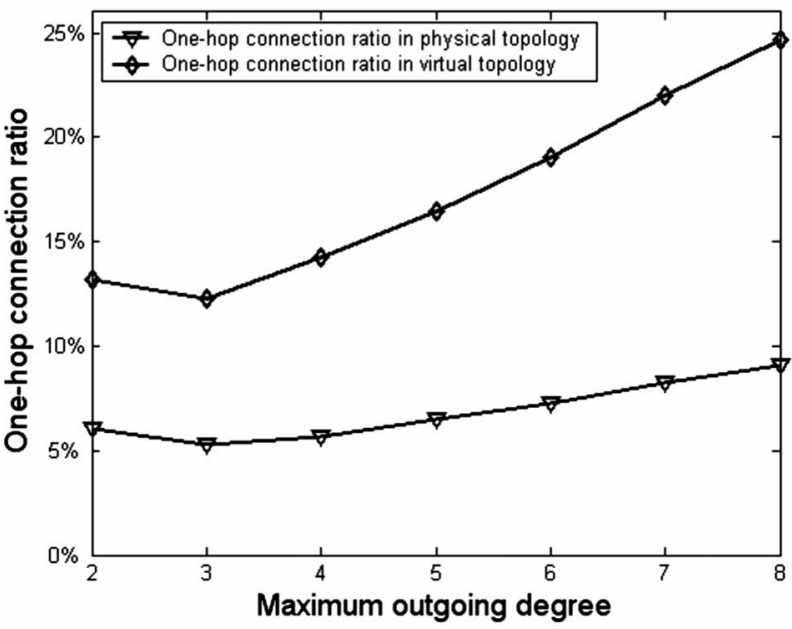

(c) One-hop connection ratio.

Fig. 5. $N=50, W=10$, and trying 100 topologies for each $D$.

connectivity is small/low. This is because when the connectivity is high, the diameter and the average hop count are already very small in the physical topology. Also, the percentage of one-hop connections in the virtual topology is increased to more than twice of the physical topology. Note 


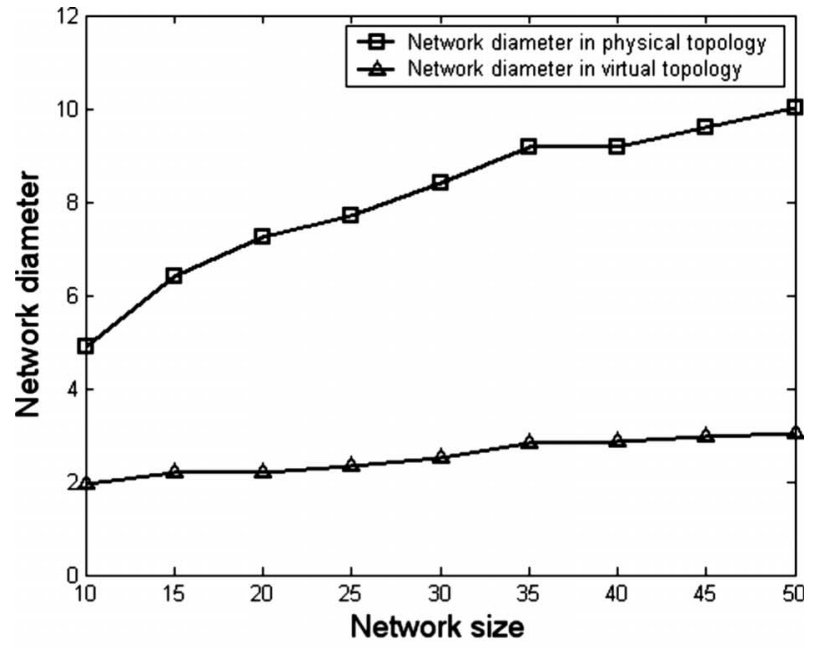

(a) Network diameter.

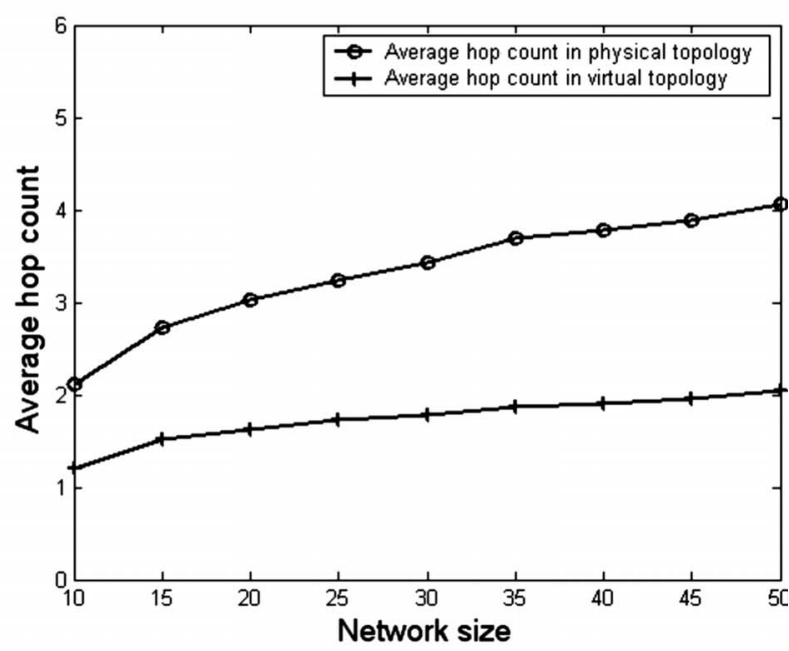

(b) Average hop count.

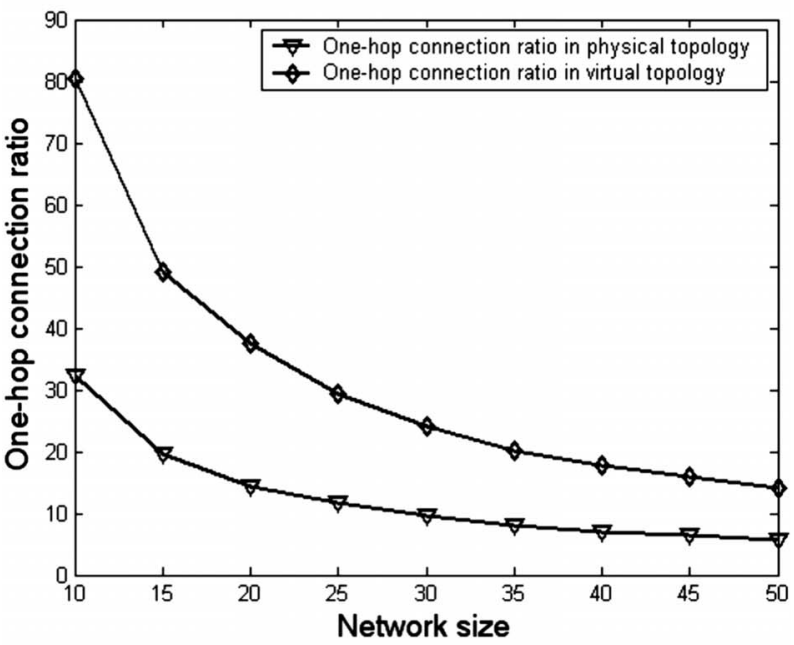

(c) One-hop connection ratio.

Fig. 6. $D=4, W=10$, and trying 100 topologies for each $N$.

that a one-hop connection implies no contention loss. In Fig. 6 , we keep $W=10$ and $D=4$, but increase $N$ from 10 to 50 with a step of 5 . For each $N$, we also average the results over 100 random topologies. As expected, both network diameter and average hop count increase with the network size, whereas the percentage of one-hop connections drops.

Figure 7 shows an LWMD design example for the National Science Foundation (NSF) network with 14 nodes and 21 links. We assume that each physical link in Fig. 7(a) is bidirectional and with a single fiber in each direction. We further assume there are $W=3$ wavelengths on each fiber. The network diameter of the physical topology is 4 . As an example, the set of dashed arrows in Fig. 7(a) gives an LSP in the physical topology, between nodes 0 and 13 and with a hop count of 4 . Figures 7(b)-7(d) show the virtual links returned by LWMD over $\lambda_{1}-\lambda_{3}$, respectively. We separate them into three figures for clarity, although the virtual topology consists of all the virtual links in Figs. 7(b)-7(d). For comparison, we use two solid arrows in Fig. 7(a) to indicate an LSP between nodes 0 and 13 in the virtual topology. Particularly, the solid virtual link $0 \rightarrow 7$ in Fig. 7(a) can be over either $\lambda_{1}$ [shown in Fig. 7(b)] or $\lambda_{3}$ [shown in Fig. 7(d)], and the solid virtual link $7 \rightarrow 13$ (which takes physical path 7 $\rightarrow 8 \rightarrow 11 \rightarrow 10 \rightarrow 12 \rightarrow 13$ ) in Fig. 7 (a) is over $\lambda_{3}$ in Fig. 7(d). In fact, it can be seen that the network diameter is now reduced from 4 in the physical topology to 2 in the virtual topology. If the traffic is uniformly distributed among all node pairs, the average hop count per connection of 2.16 hops in the physical topology is reduced to 1.62 in the virtual topology, and the one-hop connection ratio is increased from $23.08 \%$ to $38.46 \%$.

\section{TRAFFIC ACCOMMODATION SCHEMES}

In this section, we extend the problem of virtual topology design to provision enough wavelengths for a given traffic matrix $\boldsymbol{T}=\left\{t_{i j}\right\}$, where $t_{i j}$ is the number of lightpaths required to accommodate the traffic from node $i$ to node $j$. The bandwidth of each lightpath equals the capacity of a single wavelength. The problem can be formulated as follows. For a given traffic matrix $\boldsymbol{T}$, find a virtual topology that meets a predetermined network diameter requirement, such that all the requests in $\boldsymbol{T}$ can be accommodated using the least amount of network resources (i.e., total bandwidth or wavelengths).

We adopt a two-step approach. First, LWMD is used to design a virtual topology to meet the network diameter requirement using just enough wavelengths. Then, based on the virtual topology returned by LWMD, we focus on accommodating $\boldsymbol{T}$ with another set of wavelengths. Note that a request $t_{i j} \in \boldsymbol{T}$ may be split to take multiple routes. This can be achieved using an optical label switching (OLS) mechanism [28].

Figure 8(a) shows a simple physical network topology. To reduce its network diameter from 5 to 3, LWMD needs 2 wavelengths to obtain the virtual topology in Fig. 8(b), where virtual links $0 \rightarrow 2,2 \rightarrow 4$, and $4 \rightarrow 0$ (over $\lambda_{2}$ ) take physical paths $0 \rightarrow 1 \rightarrow 2,2 \rightarrow 3 \rightarrow 4$, and $4 \rightarrow 5 \rightarrow 0$, respectively. Based on the virtual topology and a given traffic matrix [e.g., Eq. (21)], we can use either a shortest-path-based scheme or a diameter-confined scheme for traffic accommodation. The shortest-path-based scheme aims at minimizing the total amount of bandwidth (i.e., wavelength links) required, and the diameter-confined scheme tries to minimize 


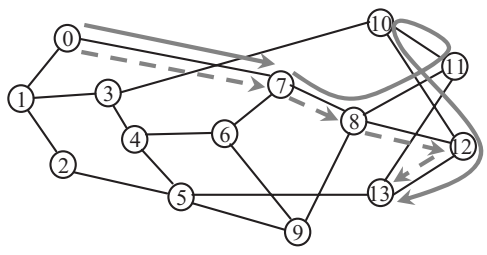

(a) NSF network with 14 nodes and 21 links (physical topology).

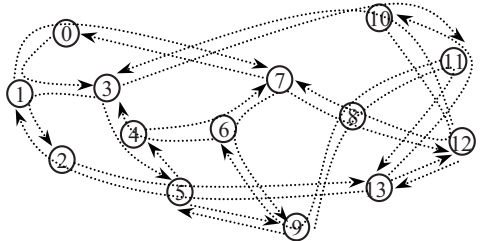

(c) Virtual links over $\lambda_{2}$

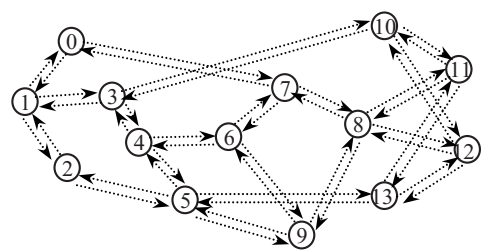

(b) $\lambda_{1}$ is used to initialize $\boldsymbol{V}$ to the same as the physical topology.

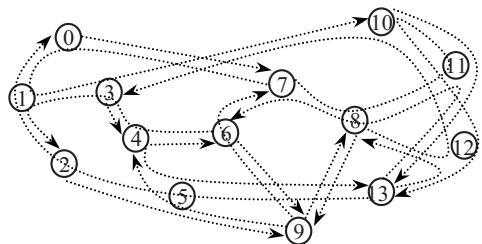

(d) Virtual links over $\lambda_{3}$.

Fig. 7. LWMD design for the NSF network with $W=3$.

the total number of wavelengths required.

$$
\boldsymbol{T}=\left\{t_{i j}\right\}=\left[\begin{array}{cccccc}
0 & 1 & 1 & 6 & 0 & 0 \\
0 & 0 & 1 & 0 & 0 & 0 \\
0 & 0 & 0 & 0 & 2 & 0 \\
0 & 0 & 0 & 0 & 2 & 0 \\
2 & 0 & 0 & 0 & 0 & 1 \\
1 & 0 & 0 & 0 & 0 & 0
\end{array}\right] .
$$

\section{A. Shortest-Path-Based Scheme}

With the shortest-path-based traffic accommodation scheme, we first route $\boldsymbol{T}=\left\{t_{i j}\right\}$ according to the shortest paths in the virtual topology and add enough wavelengths to each virtual link along the lightpaths to accommodate all the requests. The number of required wavelengths on each virtual link can thus be calculated. Based on the virtual topology in Fig. 8(b) and the traffic matrix in Eq. (21), the number next to each link in Fig. 8(c) gives the required number of wavelengths on that virtual link. For multiple parallel virtual links, such as the two virtual links $0 \rightarrow 3$ in Figs. 8(b) and 8(c) (over $\lambda_{1}$ and $\lambda_{2}$, respectively), we equally

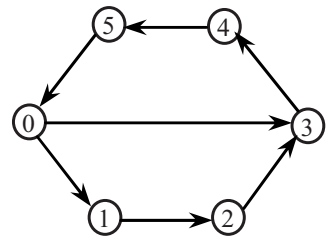

(a) Physical topology

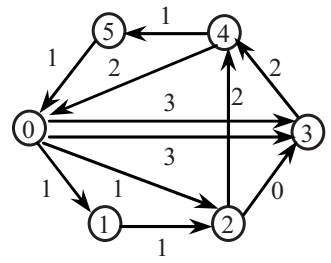

(c) Shortest path traffic mapping in the virtual topology

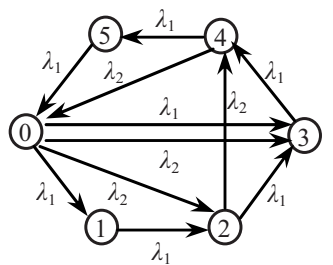

(b) Virtual topology

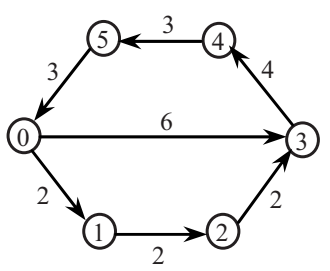

(d) NWR $=6$ in the physical topology
Fig. 8. Shortest-path-based scheme. divide the traffic load to each of them. Then, we map each virtual link back to the physical topology, together with the mapping of the wavelength requirement on the virtual link. The result is given in Fig. 8(d). As an example, the virtual link $0 \rightarrow 2$ with a demand of one wavelength is mapped to the physical links $0 \rightarrow 1$ and $1 \rightarrow 2$. Together with the original demand of one wavelength on each link, the wavelength requirement on both links $0 \rightarrow 1$ and $1 \rightarrow 2$ becomes two.

Define the network wavelength requirement (NWR) as the number of required wavelengths on the most heavily loaded link. From Fig. 8(d), the most heavily loaded physical link is $0 \rightarrow 3$, and thus the NWR of the physical topology is 6 .

\section{B. Diameter-Confined Scheme}

In the shortest-path-based scheme, the hop count is minimized, but the resulting NWR tends to be large. We can trade the average hop count for a smaller NWR, while keeping the network diameter unchanged in the virtual topology. This can be achieved by extending the Min-hops algorithm [29] to the virtual topology returned by LWMD. With the primary objective of minimizing the NWR and the secondary objective of reducing the average hop count, Min-hops is an efficient static routing algorithm. It first assigns the shortest path to all the requests and then invokes an iterative rerouting process to reduce the NWR. In each rerouting, one lightpath is routed away from the most heavily loaded link. This reduces the NWR.

The original Min-hops algorithm [29] is designed for routing in a physical topology. We extend it to the virtual topology together with an additional constraint: the hop count of any rerouted request should not exceed the target network diameter. With this constraint, although the hop count for some requests may be increased due to rerouting, we can still keep a confined network diameter in the virtual topology.

Figure 9 shows how the diameter-confined traffic accommodation scheme works. We again consider the network in Fig. 9 with $\boldsymbol{T}$ in Eq. (21). Based on Min-hops [29], the initial shortest path assignment in the virtual topology is given in Fig. 9(a) [same as Fig. 8(c)]. The two virtual links $0 \rightarrow 3$ (one dotted, one solid) carry the heaviest load of three lightpaths. Thus, in Fig. 9(a), we first reroute one lightpath from the 


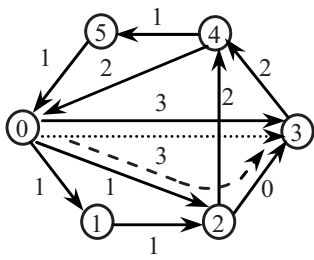

(a) Reroute the dotted $0 \rightarrow 3$

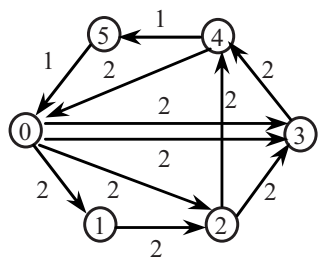

(c) Diameter confined traffic mapping in the virtual topology

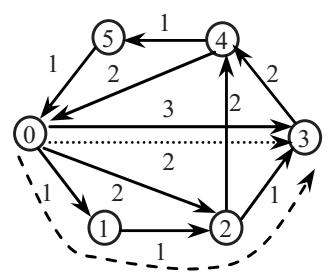

(b) Reroute the solid $0 \rightarrow 3$

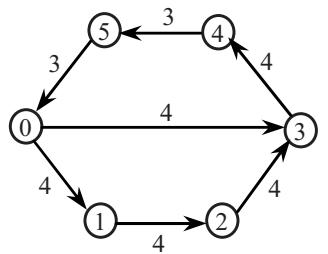

(d) NWR $=4$ in the physical topology
Fig. 9. Diameter-confined scheme.

dotted virtual link $0 \rightarrow 3$ to the dashed path $0 \rightarrow 2 \rightarrow 3$. The result of this rerouting is given in Fig. 9(b). Now the solid virtual link $0 \rightarrow 3$ carries the heaviest load. At this point, it is not suitable to reroute any lightpath from $0 \rightarrow 3$ to $0 \rightarrow 2$ $\rightarrow 3$ again because this cannot further reduce the NWR of the virtual topology. Instead, one lightpath is rerouted from the solid $0 \rightarrow 3$ to the dashed path $0 \rightarrow 1 \rightarrow 2 \rightarrow 3$ in Fig. 9(b). Note that the hop counts of the two rerouted requests do not exceed a diameter of 3. Figure 9(c) shows the traffic mapping in the virtual topology after rerouting. Finally, we map each virtual link and its wavelength requirement back to the physical topology in Fig. 9(d). We can see that the NWR of the physical topology is reduced from 6 in Fig. 8(d) to 4 in Fig. 9(d).

It is interesting to note that there is a strong correlation between load balancing in the virtual topology and that in the physical topology. Accordingly, when the load in the virtual topology is balanced using Min-hops, the load in the physical topology also spreads out to render a smaller NWR.

\section{CONCLUSION AND FUTURE WORK}

Minimizing network diameter and average hop count is very important in improving the network performance, and it can be achieved by virtual topology design. In this paper, we first formulated an ILP for optimal design of virtual topologies to minimize a linear weighted sum of network diameter and average hop count. To provide scalable designs in engineering with a short running time, we also proposed a fast and efficient heuristic LWMD, which not only minimizes network diameter and average hop count but also increases the number of one-hop connections to more than twice of that in the physical topology. Based on the virtual topology obtained, two traffic accommodation schemes (i.e., the shortest-path-based scheme and the diameter-confined scheme) were proposed to provision wavelengths for a given traffic matrix. The shortest-path-based scheme minimizes the total number of wavelength links required, whereas the diameter-confined scheme minimizes the NWR without increasing the network diameter of the virtual topology.
Future work may consider performance evaluations of a particular switching network (such as OBS or OPS) based on our virtual topology design. Compared with conventional networks without virtual topology design, our proposed scheme may need more wavelengths to minimize the network diameter and average hop count and meanwhile accommodate the same traffic. If both the traffic and the number of wavelengths on each fiber link are given, it is interesting to ask whether a better delay and throughput performance can be achieved merely by virtual topology design. Besides, it is also interesting to jointly consider virtual topology design and traffic accommodation, where minimizing the average hop count of connections is weighted by a given traffic matrix.

\section{ACKNOWLEDGMENTS}

This work was supported by the Seed Funding Program for Basic Research of The University of Hong Kong (200911159103) and a Natural Sciences and Engineering Research Council of Canada (NSERC) PDF grant.

\section{REFERENCES}

[1] L. Guo, J. Cao, H. Yu, and L. Li, "Path-based routing provisioning with mixed shared protection in WDM mesh networks," $J$. Lightwave Technol., vol. 24, no. 3, pp. 1129-1141, Mar. 2006.

[2] D. Z. Chen, G. Wellbrock, S. J. Penticost, D. Patel, C. Rasmussen, M. C. Childers, X. Yang, and M. Y. Frankel, "World's first 40 Gbps overlay on a field-deployed, $10 \mathrm{Gbps}$, mixed-fiber, 1200 km, ultra long-haul system," in Optical Fiber Communication Conf. and Expo. and the Nat. Fiber Optic Engineers Conf., 2005, vol. 2, paper OTuH4.

[3] G. X. Shen and R. S. Tucker, "Sparse traffic grooming in translucent optical networks," J. Lightwave Technol., vol. 27, no. 20, pp. 4471-4479, Oct. 2009.

[4] C. S. Xin, "Resource planning for dynamic traffic grooming in WDM optical networks," J. Lightwave Technol., vol. 27, no. 5-8, pp. 817-824, Apr. 2009.

[5] Q. D. Ho and M. S. Lee, "A zone-based approach for scalable dynamic traffic grooming in large WDM mesh networks," $J$. Lightwave Technol., vol. 25, no. 1, pp. 261-270, Jan. 2007.

[6] L. Guo, X. Wang, J. Cao, and L. Li, "Traffic recovery time constrained shared sub-path protection algorithm in survivable WDM networks," Comput. Netw., vol. 52, no. 7, pp. 1492-1505, May 2008.

[7] C. Qiao and M. Yoo, "Optical burst switching (OBS)—a new paradigm for an optical internet," J. High Speed Networks, vol. 8, no. 1, pp. 69-84, Jan. 1999.

[8] J. Li, C. Qiao, J. Xu, and D. Xu, "Maximizing throughput for optical burst switching networks," IEEE/ACM Trans. Netw., vol. 15, no. 5, pp. 1163-1176, Oct. 2007.

[9] Y. Chen, X. Yu, and C. Qiao, "Optical burst switching (OBS): a new area of research in optical networking," IEEE Network, vol. 18, no. 3, pp. 16-23, Jan. 2004.

[10] J. P. Jue and V. M. Vokkarane, Optical Burst Switched Networks. Springer, 2005.

[11] X. Huang, Q. She, T. Zhang, K. Lu, and J. P. Jue, "Modeling and performance analysis of small group multicast with deflection routing in optical burst switched networks," IEEE J. Sel. Areas Commun., vol. 26, no. 3, pp. 74-86, Apr. 2008.

[12] B. Chen and J. P. Wang, "Hybrid switching and p-routing for optical burst switching networks," IEEE J. Sel. Areas Commun., vol. 21, no. 7, pp. 1071-1080, Sept. 2003.

[13] K. Ross, N. Bambos, K. Kumaran, I. Saniee, and I. Widjaja, "Scheduling bursts in time-domain wavelength interleaved 
networks," IEEE J. Sel. Areas Commun., vol. 21, no. 9, pp. 1441-1451, Nov. 2003.

[14] J. P. Jue, W.-H. Yang, Y.-C. Kim, and Q. Zhang, "Optical packet and burst switched networks: a review," IET Commun., vol. 3, no. 3, pp. 334-352, Mar. 2009.

[15] T. S. El-Bawab and J. Shin, "Optical packet switching in core networks: between vision and reality," IEEE Commun. Mag., vol. 40, no. 9, pp. 60-65, Sept. 2002.

[16] B. Wu, K. L. Yeung, M. Hamdi, and X. Li, "Minimizing internal speedup for performance guaranteed switches with optical fabrics," IEEE / ACM Trans. Netw., vol. 17, no. 2, pp. 632-645, Apr. 2009.

[17] B. Wu, K. L. Yeung, P.-H. Ho, and X. H. Jiang, "Minimum delay scheduling for performance guaranteed switches with optical fabrics," J. Lightwave Technol., vol. 27, no. 16, pp. 3453-3465, Aug. 2009.

[18] A. S. Arora, S. Subramaniam, and H.-A. Choi, "Logical topology design for linear and ring optical networks," IEEE J. Sel. Areas Commun., vol. 20, pp. 62-74, Jan. 2002.

[19] I. Chlamtac, A. Ganz, and G. Karmi, "Lightpath communications: an approach to high bandwidth optical WAN's," IEEE Trans. Commun., vol. 40, pp. 1171-1182, July 1992.

[20] Z. Zhang and A. S. Acampora, "A heuristic wavelength assignment algorithm for multihop WDM networks with wavelength routing and wavelength re-use," IEEE/ACM Trans. Netw., vol. 3, pp. 281-288, June 1995.

[21] N. Wauters and P. Demeester, "Design of the optical path layer in multi-wavelength cross-connected networks," IEEE J. Sel. Areas Commun., vol. 14, pp. 881-892, June 1996.

[22] R. Ramaswami and K. N. Sivarajan, "Design of logical topologies for wavelength-routed optical networks," IEEE J. Sel. Areas Commun., vol. 14, pp. 840-851, June 1996.

[23] P. Pavon-Marino, R. Aparicio-Pardo, B. Garcia-Manrubia, and N. Skorin-Kapov, "Virtual topology design and flow routing in optical networks under multihour traffic demand," Photonic Network Commun., vol. 19, no. 1, pp. 42-54, Aug. 2009.

[24] F. E. El-Khamy, M. Nasr, H. M. H. Shalaby, and H. T. Mouftah, "The performance for heuristic algorithms for virtual topology design in all-optical WDM networks," in 11th Int. Conf. on Transparent Optical Networks (ICTON 2009), 2009, pp. 6467.

[25] P. Van Hentenryck and Y. Vergados, "Traveling tournament scheduling: a systematic evaluation of simulated annealing," in 3rd Int. Conf. on Integration of AI and OR Techniques in Constraint Programming for Combinatorial Optimization Problems, 2006, vol. 3990, pp. 228-243.

[26] R. K. Ahuja, T. L. Magnanti, and J. B. Orlin, Network Flows: Theory, Algorithms, and Applications. Upper Saddle River, NJ: Prentice-Hall, 1993.

[27] www.eee.hku.hk/ kyeung/vtd.c.
[28] F. Xue and S. J. B. Yoo, "High-capacity multiservice optical label switching for the next-generation Internet," IEEE Commun. Mag., vol. 42, no. 5, pp. S16-S22, May 2004.

[29] H. V. Madhyastha and N. Balakrishnan, "An efficient algorithm for virtual-wavelength-path routing minimizing average number of hops," IEEE J. Sel. Areas Commun., vol. 21, no. 9, pp. 1433-1440, Nov. 2003.

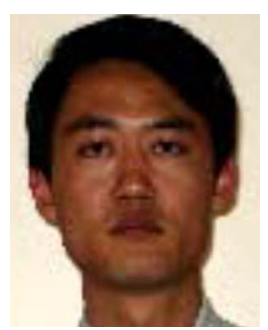

Bin Wu received the B.Eng. degree from Zhe Jiang University, Hangzhou, China, in 1993; the M.Eng. degree from the University of Electronic Science and Technology of China, Chengdu, China, in 1996; and the $\mathrm{Ph} . \mathrm{D}$. degree from the University of Hong Kong, Hong Kong, in 2007. During 19972001 , he served as the department manager of TI-Huawei DSP co-lab at Huawei Tech. Co. Ltd., Shenzhen, China. He is currently a Postdoctoral Research Fellow at the University of Waterloo, Waterloo, Canada.

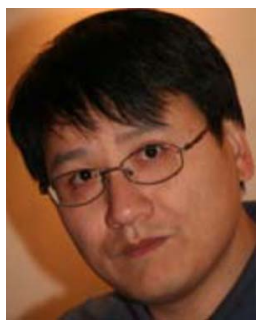

Kwan L. Yeung was born in 1969. He received his B.Eng. and Ph.D. degrees in information engineering from the Chinese University of Hong Kong in 1992 and 1995, respectively. He joined the Department of Electrical and Electronic Engineering, The University of Hong Kong, in July 2000, where he is currently a Professor. His research interests include next-generation Internet, packet switch/router design, alloptical networks, and wireless data

networks.

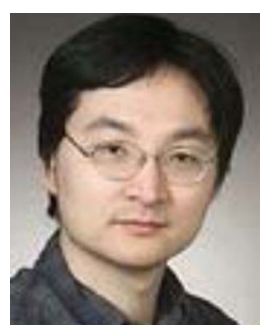

Pin-Han Ho received his B.Sc. and M.Sc. degrees from the Electrical and Computer Engineering Department of National Taiwan University, in 1993 and 1995, respectively. He started his Ph.D. studies in 2000 at Queen's University, Kingston, Ontario, Canada, focusing on optical communications systems, survivable networking, and QoS routing problems. He finished his Ph.D. in 2002, and joined the Electrical and Computer Engineering Department at the University of Waterloo as an Assistant Professor in the same year. $\mathrm{He}$ is the author/co-author of more than 100 refereed technical papers and book chapters and the co-author of a book on optical networking and survivability. He is the recipient of the Distinguished Research Excellence Award in the ECE Department at the University of Waterloo, the Early Researcher Award in 2005, the Best Paper Award at SPECTS '02 and the ICC '05 Optical Networking Symposium, and the Outstanding Paper Award in HPSR '02. 